\title{
Article
}

\section{Loop Formation of a Flexible Polymer with Two Random Reactive Sites}

Yu-Jane Sheng, Pei-Hsien Hsu, Jeff Z. Y. Chen, and Heng-Kwong Tsao

Macromolecules, 2004, 37 (24), 9257-9263 • DOI: 10.1021/ma049388z

Downloaded from http://pubs.acs.org on November 24, 2008

\section{More About This Article}

Additional resources and features associated with this article are available within the HTML version:

- $\quad$ Supporting Information

- $\quad$ Links to the 2 articles that cite this article, as of the time of this article download

- $\quad$ Access to high resolution figures

- $\quad$ Links to articles and content related to this article

- $\quad$ Copyright permission to reproduce figures and/or text from this article

\section{View the Full Text HTML}




\title{
Loop Formation of a Flexible Polymer with Two Random Reactive Sites
}

\author{
Yu-Jane Sheng, ${ }^{* \dagger}$ Pei-Hsien Hsu, ${ }^{\dagger}$ Jeff Z. Y. Chen, ${ }^{\ddagger}$ and Heng-Kwong Tsao ${ }^{*, \S}$ \\ Department of Chemical Engineering, National Taiwan University, Taipei, Taiwan, 106, R.O.C.; \\ Department of Physics, University of Waterloo, Waterloo, Ontario, Canada N2L 3G1; and Department \\ of Chemical and Materials Engineering, National Central University, Jhongli, Taiwan 320, R.O.C.
}

Received March 28, 2004; Revised Manuscript Received September 15, 2004

\begin{abstract}
The dynamics of loop formation is investigated for a flexible polymer owing to the reversible intrachain reaction. The polymer is made of $N$ hard spheres tethered by inextensible bonds, and the two reactive sites with binding energy $-\epsilon$ are randomly located. The coil-to-loop crossover is characterized by the probability curve which depicts the variation of the open-state probability with temperature. The midpoint temperature $\left(\beta_{\mathrm{m}}{ }^{-1}\right)$ is related to the conformational entropy loss $\Delta S$ from coil to loop states. It is found that $\beta_{\mathrm{m}} \epsilon=\Delta S / k_{\mathrm{B}}=\ln N^{\alpha}+G$, and the loop can be classified into three types: (i) end-to-end, $\alpha \simeq 1.98$; (ii) end-to-interior, $\alpha \simeq 2.16$; and (iii) interior-to-interior, $\alpha \simeq 2.48$. The constant $G$ varies with locations of the reactive sites. The kinetic rate constants can be well depicted by the Arrhenius kinetics with free energy barriers in agreement with crossover thermodynamics. Although the end-to-end loop is most easily formed for long enough polymers, the interior-to-interior loop is preferred for short chains. This consequence indicates that biopolymers may utilize loop types and contour distance between reactive sites to control the probability of loop formation.
\end{abstract}

\section{Introduction}

An associating polymer consists of a small number of active groups, which are attached at various points along the polymer backbone. These groups, such as ionic and hydrophobic segments or complementary base pairs, may react with one another if the chain motion brings them into close proximity of one another. The intramolecular binding hence leads to loop formation. The simplest example of associating chains is a telechelic polymer, which is a water-soluble chain with relatively small associating groups at the ends, for instance, poly(ethylene oxide) with end-capped $\mathrm{C}_{16}-\mathrm{C}_{18}$ alkanes. Recently, telechelic biopolymers have attracted much attention owing to the particular importance of polymer loop in biology. A typical example is a singlestranded DNA or RNA, which is purposefully designed to be made of a homogeneous sequence such as poly(A) with a few complementary bases at both ends. ${ }^{1}$

Polymer looping is widely used in nature. In particular, DNA looping induced by protein is well documented in regulating all aspects of DNA metabolism, including transcription, replication, and recombination. ${ }^{2}$ In general, this protein consists of two connected domains: a DNA-binding domain that binds to the well-separated operator sites and a dimerization domain with two proteins that bind cooperatively as a dimer. ${ }^{3}$ The endbound and internally bound DNA looping are both commonly observed. ${ }^{4}$ When the polymer looping involves large loop sizes, e.g., several thousand base paring, the loop formation causes a significant entropy loss which has to be overcome by the binding energy released on loop closure. Despite considerable theoretical efforts in studying the end-to-end loop formation, ${ }^{5,6}$ there are relatively few results concerning the end-to-interior and interior-to-interior loop formation.

\footnotetext{
National Taiwan University.

ঊ University of Waterloo.

$\S$ National Central University.

* Corresponding authors. E-mail: yjsheng@ccms.ntu.edu.tw; hktsao@cc.ncu.edu.tw.
}

In the present study, we investigate the coil-to-loop crossover and quantify the entropy loss associated with loop formation for a flexible polymer with two random reactive sites. The outline of this paper is as follows. First, we write down the partition function associated with a single hard-sphere chain based on the two-state scenario (coil and loop states). Then, the variation of the probability of the coil state and the heat capacity with the temperature can be derived. We prove that the inverse midpoint temperature of the coil-state probability curve is proportional to the entropy loss associated with loop formation. Three types of loop formation are considered: (i) end-to-end, (ii) end-to-interior, and (iii) interior-to-interior. The results of Monte Carlo simulations confirm our theory and provide the exponent associated with chain length dependence and the entropy constant which varies with the contour distances between reactive sites.

\section{Theory}

Consider a flexible chain of total length $L$. The reactive groups are located at beads " $p$ " and " $q$ ". That is, the two reactive groups are positioned at distance $a$ and $b$, respectively, from the chain ends as illustrated in Figure 1. We define $l \equiv L-(a+b)$ to be the contour distance between the active groups. The partition function associated with such a polymer depends on the location of the reactive sites and is given by

$$
\mathcal{A}\left(\frac{a}{l}, \frac{b}{l}, N\right)=\sum_{i} g_{i} \exp \left(-\beta \epsilon_{i}\right)
$$

where $g_{i}$ denotes the degeneracy associated with the state $i$ of internal energy $\epsilon_{i}$ and $\beta$ is the inverse temperature.

When the two reactive sites are in close proximity, the reversible reaction occurs and the binding energy is $-\epsilon$. Such conformations are thus identified as the loop state $(l)$. Otherwise, the conformations are regarded as the coil state $(o)$. As a result, the partition function 


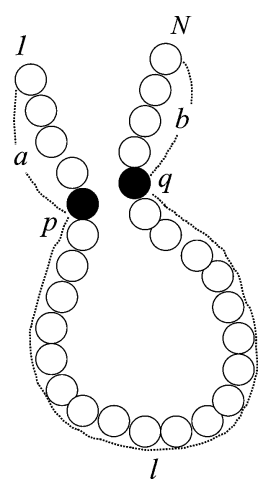

Figure 1. Schematic of a hard-sphere chain with $N$ beads. The two reactive groups locate at beads $p$ and $q$.

can be divided into two parts

$$
z=z_{0}+z_{l}
$$

where $\mathscr{z}_{o}=g_{o}$ and $\mathscr{Z}_{l}=g_{l} \exp (\beta \epsilon)$. The degeneracy $g_{i}$ varies with the spatial range associated with the reversible reaction. In terms of the partition function, the probability of the coil state is simply

$$
P_{o}\left(T ; \frac{a}{l}, \frac{b}{l}\right)=\frac{\mathscr{Z}_{o}}{\mathcal{Z}}=\frac{1}{1+\frac{g_{l}}{g_{o}} \mathrm{e}^{\beta \epsilon}}=\frac{1}{1+\mathrm{e}^{\left(\beta-\beta_{\mathrm{m}}\right) \epsilon}}
$$

The probability curve denotes the variation of the coilstate probability with temperature. We define the midpoint temperature $T_{\mathrm{m}}$ (or $\beta_{\mathrm{m}}$ ) as the temperature where the probabilities of coil and loop states are equal.

The physical significance of the midpoint temperature can be manifested through the conformational entropy. On the basis of the two-state scenario, the entropy loss from the open to closed states is obtained from the partition function, eq 1

$$
\Delta S\left(\frac{a}{l}, \frac{b}{l}\right)=S_{o}-S_{l}
$$

with $S_{i}=k_{\mathrm{B}} \ln g_{i}$. In accordance with eq 3 , the relation between the midpoint temperature and the entropy loss is thus given by

$$
\beta_{\mathrm{m}} \epsilon=\ln \frac{g_{o}}{g_{l}}=\frac{\Delta S}{k_{\mathrm{B}}}
$$

This result states that at the midpoint temperature the entropy loss is exactly compensated by the energy gain. Equation 4 presents an important consequence that one is able to evaluate the entropy change (or the probability of finding two points in close proximity) of a polymer chain from the coil-state probability curve.

\section{Monte Carlo Simulation}

In this paper, we aim to study polymers with only hard-core excluded-volume effects but without other extra interaction energy such as attractions among monomers and solvent. The model associating chain is made of $N$ hard spheres of diameter $\sigma$ with attractive beads located at $p$ and $q$. This polymer is a freely jointed chain in continuous space. The interactions between the bonded beads are through the infinite deep square-well potentials

$$
U_{i, i+1}= \begin{cases}\infty & r<\sigma \\ 0 & \sigma \leq r<\xi \sigma \\ \infty & r \geq \xi \sigma\end{cases}
$$

where $\xi=1.2$. Bond crossing (phantom chain) can be prevented by such a choice. For this model associating chain, the interaction between attractive beads $p$ and $q$ is represented by a standard square-well potential

$$
U_{p, q}= \begin{cases}\infty & r<\sigma \\ -\epsilon & \sigma \leq r<\lambda \sigma \\ 0 & r \geq \lambda \sigma\end{cases}
$$

where $\lambda=1.2$. Without the loss of generality, we assume the binding energy $\epsilon=15$.

In this work, Monte Carlo (MC) simulations were performed to study both thermodynamics and kinetics associated with loop-to-coil crossover. Note that MC simulation is generally not the method of choice for investigating kinetics. Nonetheless, because the actual dynamics has reached thermodynamic equilibrium, it is justified to estimate the reaction constants of the kinetics by MC method. The chain is identified as in the "coil" state when the attractive beads of the chain are not within square-well interaction regimes. That is, $\left|\mathbf{r}_{p}-\mathbf{r}_{q}\right|>\lambda \sigma$. On the other hand, the "loop" state is defined as the formation of an attractive pair from two ends. That is, $\left|\mathbf{r}_{p}-\mathbf{r}_{q}\right|<\lambda \sigma$. The rate constant $k_{i, j}$ is evaluated from the inverse of the mean time $\tau_{i, j}$. It is defined as the mean period of staying at the state $i$, which jumps to the state $j$ later. The probability of the state $i$ is calculated by the total Monte Carlo steps (MCs) staying at the state $i$ to the total MCs of simulation. The detailed balance, $k_{o, l} P_{o}=k_{l, o} P_{l}$, is fulfilled in our simulations.

The systems simulated contain a single polymer chain with chain length $N$ ranging from 15 to 68 . The simulations are performed under the conditions of constant temperature and total number of beads. In the present study, the reduced temperature $T^{*}$ is varied to obtain the melting curves, which are represented by $P_{o}$ and $P_{l}$. The trial moves employed for chains of the equilibration and production process are bead displacement motions. They involve randomly picking a bead and displacing it to a new position in the vicinity of the old position. The distance away from the original position is chosen with a probability, which satisfies the condition of equal sampling of all points in the spherical shell surrounding the initial position. The new configurations resulting from this move are accepted according to the standard Metropolis acceptance criterion. Runs for the same chain length at different temperatures are performed starting with the final configuration from a previous temperature and are equilibrated for 200 million steps. Measurements for static properties such as the probabilities of open or closed states are taken over a period of 5-10 million MCs per bead. Note that the chain relaxation time defined by end-to-end autocorrelation function $(\epsilon=0)$ is about $10^{5} \mathrm{MCs}$ per bead for $N=20$.

\section{Results and Discussion}

On the basis of the two-state scenario, one is able to relate the entropy loss associated with the coil-to-loop crossover or the probability of finding two points of a 


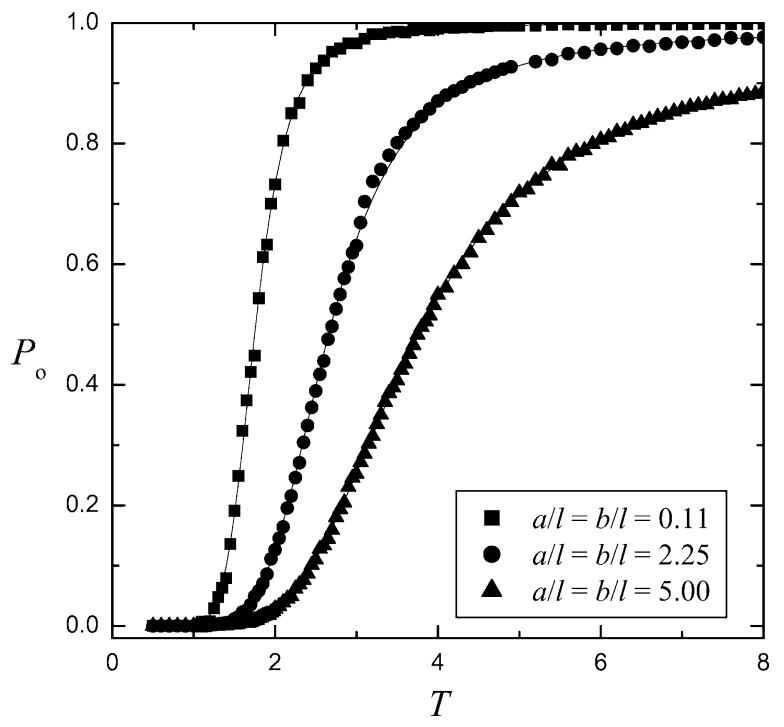

Figure 2. Open-state probability curve for different separations between reactive sites with chain length $N=24$. The MC results and theoretical curves, eq 3, are denoted by data points and solid curves, respectively.

flexible polymer in close proximity to the midpoint temperature in the open-state probability curve. Evidently, the number of open conformations varies only with the chain length, $g_{o}(N)$, while the number of loop conformations also depends on the contour distances $a / l$ and $b / l$, i.e., $g_{l}(a / l, b / l, N)$. Therefore, eq 4 shows that the midpoint temperature varies with $a / l, b / l$, and $N$. Three limiting cases can be identified: (i) end-to-end attraction $(a / l=b / l=0)$, (ii) end-to-interior attraction $(a / l=0$ and $b / l>0)$, and (iii) interior-to-interior attraction $(a / l=b / l>0)$.

We performed off-lattice Monte Carlo (MC) simulations to calculate the open-state probability curve. Figure 2 depicts a typical example of $P_{o}$ for different contour distances of two reactive sites for a given chain length $N=24$. Note that the distance ratio $a / l=b / l$ varies from much less to much greater than unity. The data points represents the MC result and can be well represented by eq 3 . The midpoint temperature is read directly from simulation data at $P_{o}=1 / 2$ or determined by fitting data of all temperature range. Both methods give the same result. As the temperature rises, the probable structure shifts from a stable loop state to a stable coil one. Moreover, the midpoint temperature of the structure grows generally with increasing the ratio of $a / l$. To compare MC results with eq 3 further, we plot $\ln \left[P_{o}^{-1}-1\right]$ against $\left(\beta-\beta_{\mathrm{m}}\right)$ for various combination of $a / l, b / l$, and $N$. All the probability curves associated with different chain lengths and contour distances of reactive sites collapse into a straight line with a slope $\epsilon$ as shown in Figure 3a. Since the heat capacity is related to the internal energy $U$ by

$$
C=\frac{\left\langle U^{2}\right\rangle-\langle U\rangle^{2}}{k_{\mathrm{B}} T^{2}}
$$

the variation of the heat capacity with temperature can be evaluated directly from eq 3

$$
\frac{C\left(\frac{a}{l}, \frac{b}{l}, N\right)}{k_{\mathrm{B}}}=(\beta \epsilon)^{2} \frac{\mathrm{e}^{\left(\beta-\beta_{\mathrm{m}}\right) \epsilon}}{\left[1+\mathrm{e}^{\left(\beta-\beta_{\mathrm{m}}\right) \epsilon}\right]^{2}}
$$
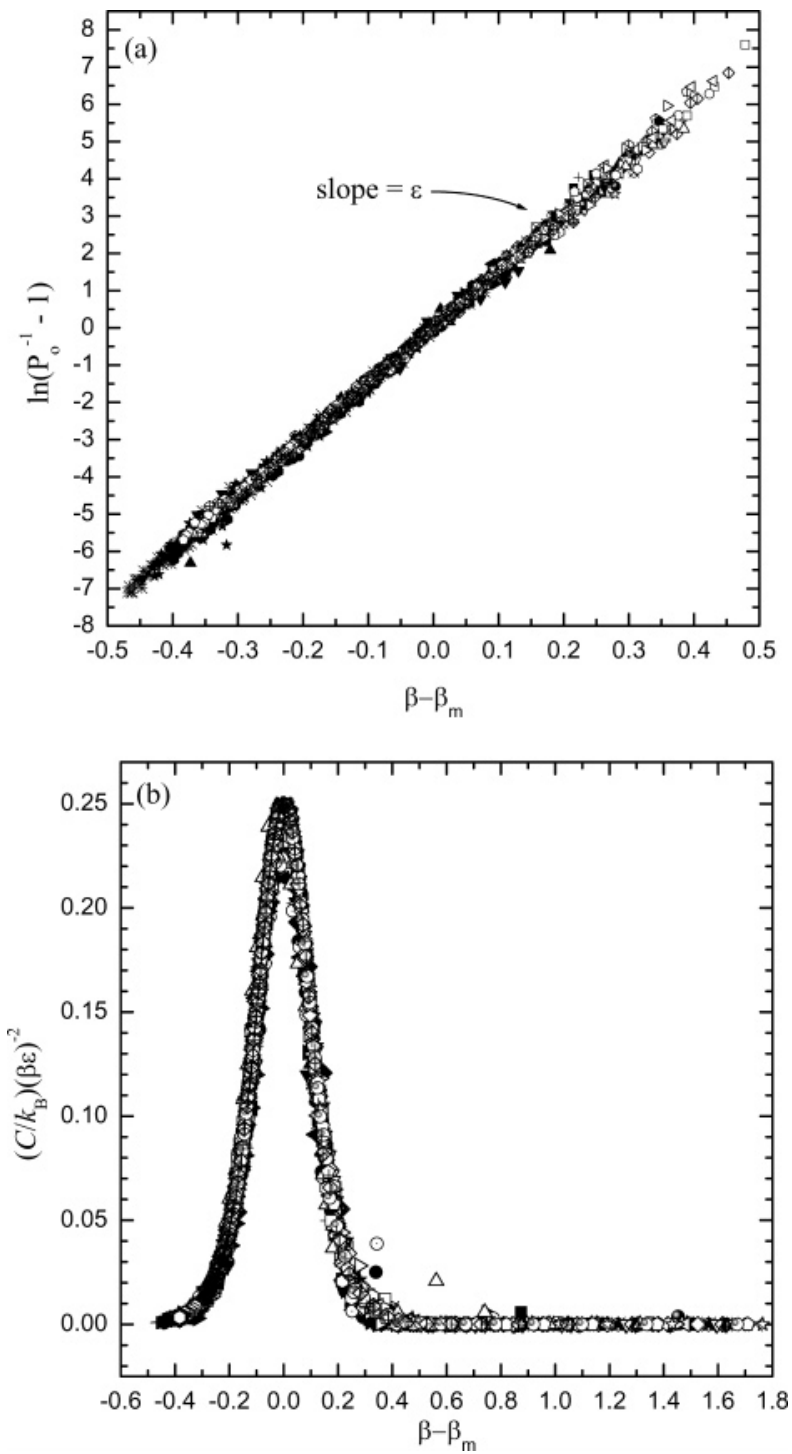

Figure 3. (a) Open-state probability curves for different chain lengths are replotted with $\ln \left(P_{o}^{-1}-1\right)$ as a function of $(\beta-$ $\beta_{\mathrm{m}}$ ) and compared to eq 3 . (b) Heat capacity curves for different chain lengths are plotted with $(C / k)(\beta \epsilon])^{-2}$ as a function of $(\beta$ $\left.-\beta_{\mathrm{m}}\right)$ and compared to eq 5 .

When we plot $\left(C / k_{\mathrm{B}}\right)(\beta \epsilon)^{-2}$ against $\left(\beta-\beta_{\mathrm{m}}\right)$ in Figure $3 \mathrm{~b}$, all data points of different combinations of $a / l, b / l$, and $N$ fall into a single curve as indicated by eq 5 . The above results confirm that thermodynamics of reversible loop formation of a flexible chain displays the same characteristics associated with coil-to-loop crossover. The dependence on chain length and contour distance between reactive sites are only manifested through the midpoint temperature.

The entropy loss from coil to loop states grows with chain length. Its scaling behavior can be realized from the probability of nearest-neighbor contacts between two reactive sites, $\left.\mathscr{A}\left|\mathbf{r}_{p}-\mathbf{r}_{q}\right| \approx O(\sigma)\right] \sim N^{-v(3+\theta)} \mathscr{G}(a / l, b / l)$. Since $\left.Z_{l} \sim Z_{0} \not \partial \sigma\right)$, one has $\Delta S / k_{\mathrm{B}}=\ln z_{0} / Z_{l} \sim v(3+\theta)$ $\ln N+G$. The correlation length exponent is $v \simeq 3 / 5$ for a good solvent, and $\theta$ is the correlation hole exponent which describes the short-distance spatial decay of the corresponding probability distribution due to the excluded-volume interaction. The short distance exponent $\theta$ depends on the locations of reactive sites, and three special cases have been calculated by field theory or simulation of self-avoiding chain on a lattice: ${ }^{7}$ (i) $\theta_{0} \approx$ 

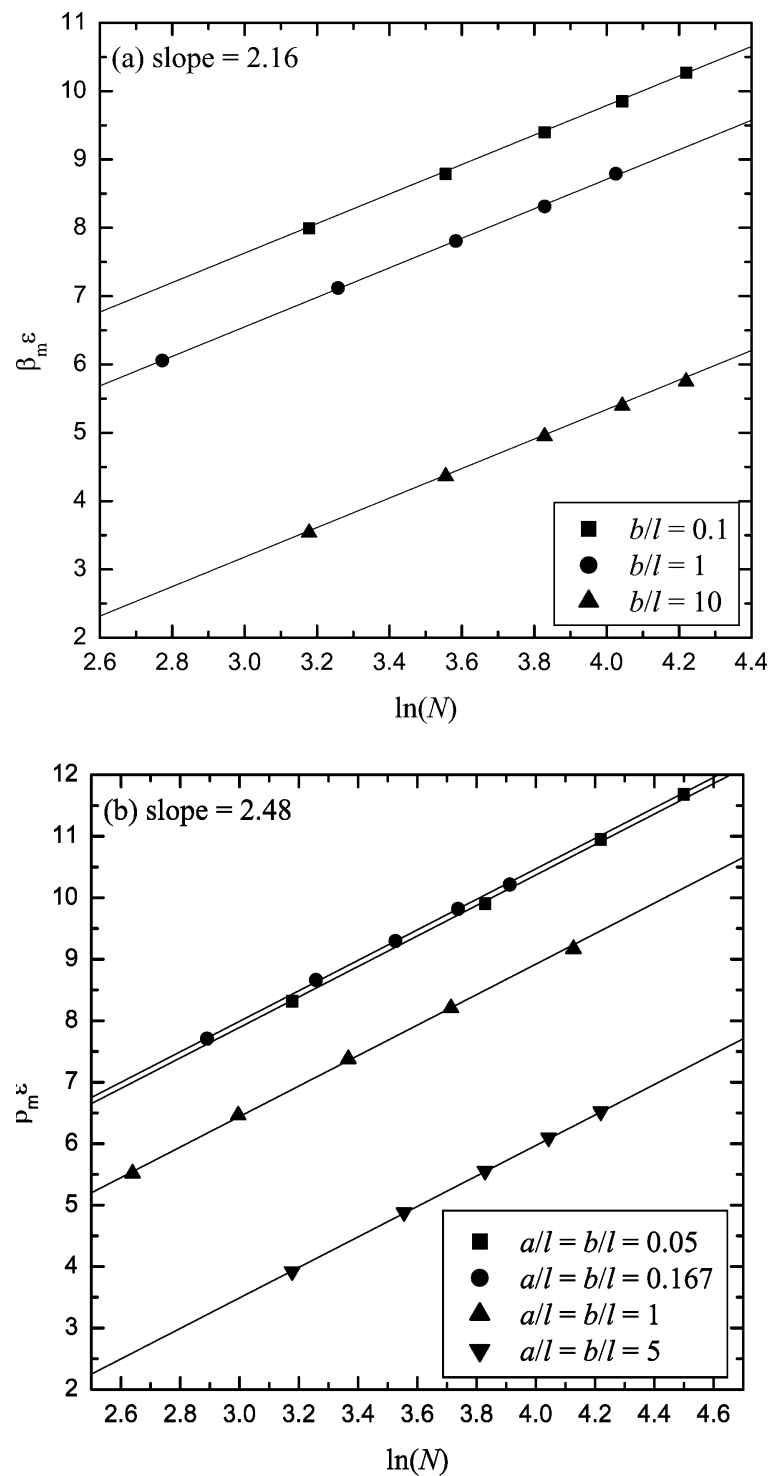

Figure 4. (a) Inverse midpoint temperature is plotted against the chain length for the end-to-interior case $(a / l=0$ and $b / l \neq$ 0 ). The straight lines denote eq 6 with $\alpha=2.16$. (b) Inverse midpoint temperature is plotted against the chain length for the interior-to-interior case $(a / l=b / l \neq 0)$. The straight lines denote eq 6 with $\alpha=2.48$.

0.28 for end-to-end contact, (ii) $\theta_{1} \approx 0.46$ for end-tointerior contact, and (iii) $\theta_{2} \approx 0.71$ for interior-to-interior contact. According to eq 4, the midpoint temperature is anticipated to follow the same scaling law. For end point-end point loop formation, it is reported ${ }^{6}$ that

$$
\beta_{\mathrm{m}} \epsilon=\alpha \ln N+G\left(\frac{a}{l}, \frac{b}{l}\right)
$$

where $\alpha_{0} \simeq 1.98$ and $a / l=b / l=0$. This result agrees quite well with $\alpha_{0}=v\left(3+\theta_{0}\right)$ with $\theta_{0} \approx 0.28$.

For end-to-interior and interior-to-interior loop formation, one anticipates that the entropy loss or midpoint temperature depends on the chain length as well as the contour distance between two reactive sites. The variation of the midpoint temperature $\beta_{\mathrm{m}} \epsilon$ with chain length $\ln N$ is depicted in Figure 4a,b. Both of them follow eq 6 for different ratios of $a / l$ and $b / l$, and we obtain $\alpha_{1} \simeq$ 2.16 for the former and $\alpha_{2} \simeq 2.48$ for the latter. In comparison with the end-to-end loop, the increment of the exponent is caused by the excluded-volume interac- tions between the loop part $(l)$ and the linear part ( $a$ and $b$ ). It is interesting to note that the short distance exponent calculated by $\theta_{i}=\alpha_{i} / v-3$ is greater than the previous reported value. ${ }^{7}$ That is, $\theta_{1}=0.67$ and $\theta_{2}=$ 1.22. The possible explanation is that terms higher than second order are required to obtain convergent results for field theory and renormalization group theory. When exact counting method was adopted to study a lattice self-avoiding walk model, the chain length was probably not long enough to obtain accurate values for $\theta_{i}{ }^{8}$ Our results indicate that when the chain length is very long, i.e., $N \gg \exp (|G| / \alpha)$, the entropy loss is largest for the formation of interior-to-interor loop and smallest for the end-to-end loop. In other words, it is relatively difficult to form a loop between two interior points for a specified chain length at a given temperature.

Since the entropy loss is logarithmically increased with the chain length, the next order term is important for relatively short polymers. The entropy constant $G$ varies only with the location of reactive sites $a / l$ and $b / l$. The validity of eq 6 can be further verified by plotting $\left(\beta_{\mathrm{m}} \epsilon-\alpha \ln N\right)$ against b/l. All data points of different chain lengths fall into a single curve as shown in Figure 5a for end-to-interior case $(a / l=0)$ and in Figure $5 \mathrm{~b}$ for interior-to-interior case $(a / l=b / l)$. Two asymptotic features are observed. When $b / l \gg 1$, the entropy constant $G$ declines linearly with $\ln (b / l)$. That is, $\mathscr{g}(b / l)$ is scaled as $(b / l)^{x}$ for $b \gg l$ with $x \simeq 1.86$ for end-to-interior loop and $x \simeq 2.24$ for interior-to-interior loop. If one considers the limiting case $a / l=0$ and $b / l \simeq$ $N$, the entropy loss in forming such an trivial loop is negligible, and from eq 6 it follows $G(0, b / l)=x \ln (b / l)$ with $x=\alpha_{1}$. Similarly, one can consider another limiting case $a / l=b / l \simeq N / 2$ and obtain $G(b / l)=x \ln (b / l)$ with $x$ $=\alpha_{2}$. From Figure 5a,b we obtain the exponent $x$ slightly smaller than the corresponding value of $\alpha$. It may be attributed to the fact that $\ln (b / l)$ is not large enough, and the next order term cannot be ignored completely. On the other hand, as $b / l \ll 1$, the entropy constant $G$ varies very slowly with decreasing $\ln (b / l)$. It is natural to expect that the value of $G$ is finite as $a / l=b / l \rightarrow 0$. Note that under the asymptotic condition both reactive sites located at polymer ends $\ln (b / l) \rightarrow-\infty$, the entropy constant becomes $G(0,0) \simeq 1.34$ and the exponent jumps to $\alpha_{0} \cdot{ }^{6}$ Therefore, the cases involving the end point of the chain correspond to singular conditions associated with $G(a / l, b / l)$. As $\ln (b / l)$ is decreased, we observe that $G$ for the end-to-interior case $\left(G_{1}\right)$ is gradually increased toward an asymptotic value about 1.20. Nevertheless, $G$ for interior-to-interior loop $\left(G_{2}\right)$ displays a maximum point at about $b / l \simeq 0.14$.

Since eq 6 must be applicable to the ideal chain as well, we may gain some insight by examining this particularly simple case. In the Gaussian chain limit, the exponents $\theta_{i}$ vanishes and one has

$$
\Delta S=3 v \ln N-3 v \ln \left(1+\frac{a}{l}+\frac{b}{l}\right)
$$

with $v=1 / 2$. Since the excluded-volume effect is not considered, all three cases merge into one. When $(a+$ $b) / l \ll 1, G \simeq 3 v(a+b) / l \rightarrow 0$. On the other hand, as $a$ $=b \gg l$, one obtains $G \simeq-3 v[\ln 2+\ln (b / l)]$. When the excluded volume is taken into account, additional exponents such as $\theta_{i}$ are involved. Nevertheless, it is reasonable to conjecture that $G=-\left[3 v+f\left(\theta_{i}\right)\right] \ln (b / l)$ for $a / l=b / l \gg 1$, as observed in Figure 5. Equation 6 clearly shows that there are three factors in determining 

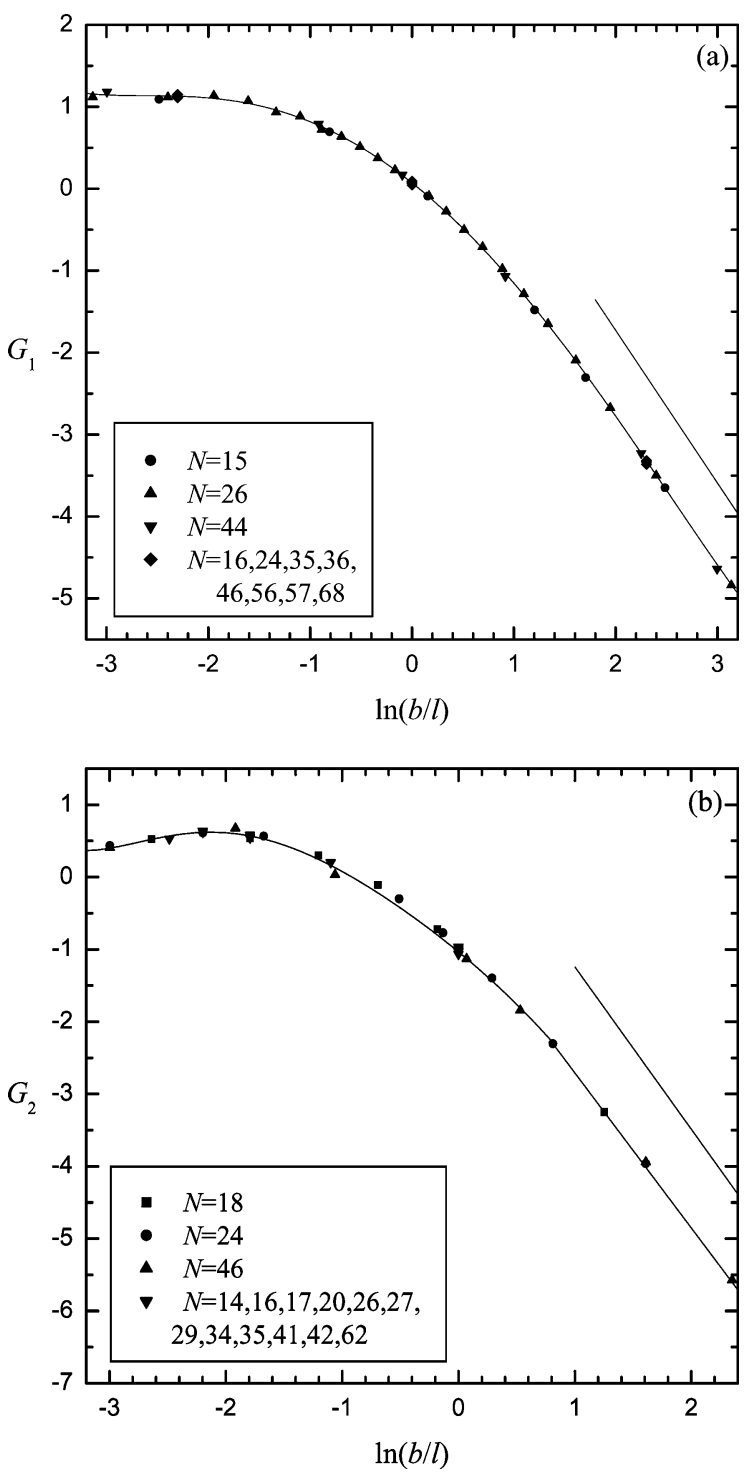

Figure 5. Entropy constant $G$ is a function of the ratio of contour distances $b / l$ at different chain lengths: (a) the endto-interior case; (b) the interior-to-interior case. The dotted line indicates the asymptotic behavior, $G \sim \ln (b / l)^{x}$ for $b / l \gg 1$.

the midpoint temperature: the loop type, the chain length, and the contour distance between reactive sites. This result indicates that in order to have higher midpoint temperature (higher probability of loop formation) the end-to-end case provides the smallest exponent $\alpha$, but the interior-to-interior case gives a smaller constant $G$ associated with large $(a+b) / l$. Figure 6 illustrates that the short contour distance may dominate over the location of reactive sites for short enough polymers. However, as the chain length is increased, the location of reactive sites becomes more important. Eventually, the end-to-end loop is more easily to formed.

Because the binding energy $\epsilon$ is comparable to the thermal energy $k_{\mathrm{B}} T$, the loop formation is reversible in the present study. As a result, the coil-to-loop crossover can be regarded as chemical equilibrium associated with a reversible reaction as well. ${ }^{6}$ The reactant and product are respectively the coil and loop states, i.e., coil $\Leftrightarrow$ loop. Note that the reaction becomes irreversible if $\beta \epsilon \rightarrow \infty$. At equilibrium, the principle of detailed balance is satisfied, and the characteristic of the kinetics can be explored by examining the rate constants evaluated from MC simulations. The rate coefficients $k_{i, j}=\tau_{i, j}{ }^{-1}$

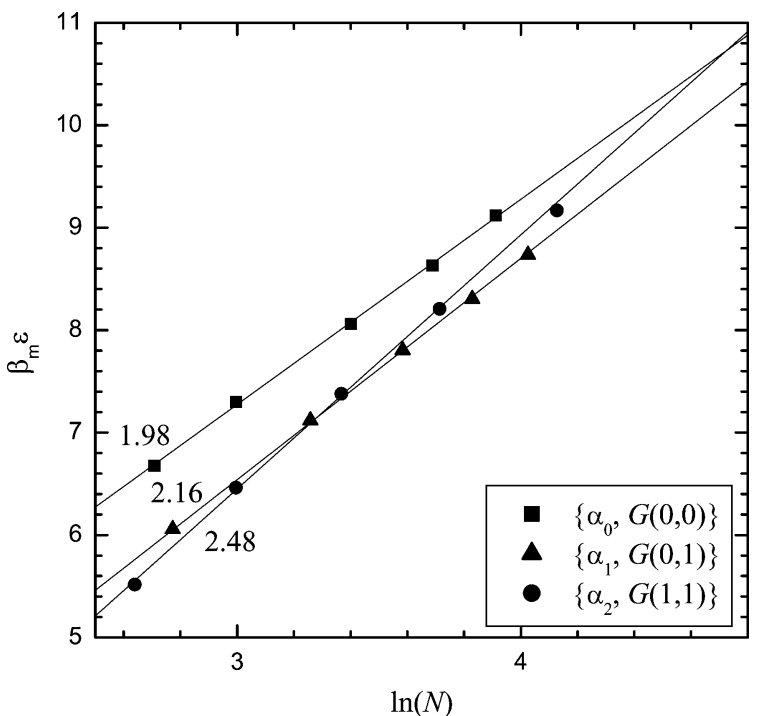

Figure 6. Variation of the midpoint temperature $\beta_{\mathrm{m}} \epsilon$ with the chain length $N$ for the three types of loop formation.

from the $i$ to $j$ state are assumed to follow the Arrhenius kinetics

$$
k_{i, j}=k_{i, j}^{0} \exp \left(-\beta F_{i, j}\right)
$$

where $F_{i, j}$ represents the free energy barrier associated with jumping from the $i$ to $j$ states. The preexponential factor $k_{i, j}^{0}$ is temperature-independent. When the conformation of a hard-sphere chain changes from the coil state to the loop one, it is natural to anticipate that the free energy barrier amounts to the entropy loss from a random coil to a loop, $\beta F_{o, l}=\left(S_{o}-S_{l}\right) / k_{\mathrm{B}}$. Consequently, $k_{o, l}$ is independent of temperature but varies with chain length. According to eq 6, one has

$$
\ln \left(\tau_{o, l} / N^{\alpha}\right)=G\left(\frac{a}{l}, \frac{b}{l}\right)+\ln k_{o, l}^{0}
$$

This result points out that $\Delta \ln \left(\tau_{o, l} / N^{\alpha}\right)=\Delta G(a / l, b / l)$. As shown in Figure 7a,b, all the rate constants computed from different chain lengths fall into a constant line with zero slope when $\ln \left(k_{o, l} l^{-1} / N^{\alpha}\right)$ is plotted against $\beta$. The difference between the intercepts of two sets of (a/l, b/l) agrees with $\Delta G$ evaluated from Figure 5a,b.

On the other hand, when the polymer conformation fluctuates from the loop state to the coil one, the free energy barrier, which has to be overcome, is simply the binding energy, $\beta F_{l, o}=\beta \epsilon$ and independent of the chain length $N$. As illustrated in Figure 8a,b, when $\ln \left(k_{l, o}{ }^{-1}\right)$ is plotted against $\beta$, all the rate coefficients calculated from different chain lengths $N$ collapse into a single line with a slope of the binding energy $\epsilon$. This consequence shows that the thermal fluctuation provides a probability of $\exp (-\beta \epsilon)$ to unbind the loop conformation regardless of the chain length. The above analysis confirms the Arrhenius characteristics of the rate constants $k_{i, j}$ associated with the coil-loop equilibrium in terms of free energy barriers.

Biomacromolecue DNA performs its biological functions with loop formation. Intuitively, one expects that short contour distance is the major factor for controlling the loop formation. However, it is known that DNA looping often involves large loop sizes of several thousand base pairs. Our simulation results show that the interplay between the loop type and the contour distance 

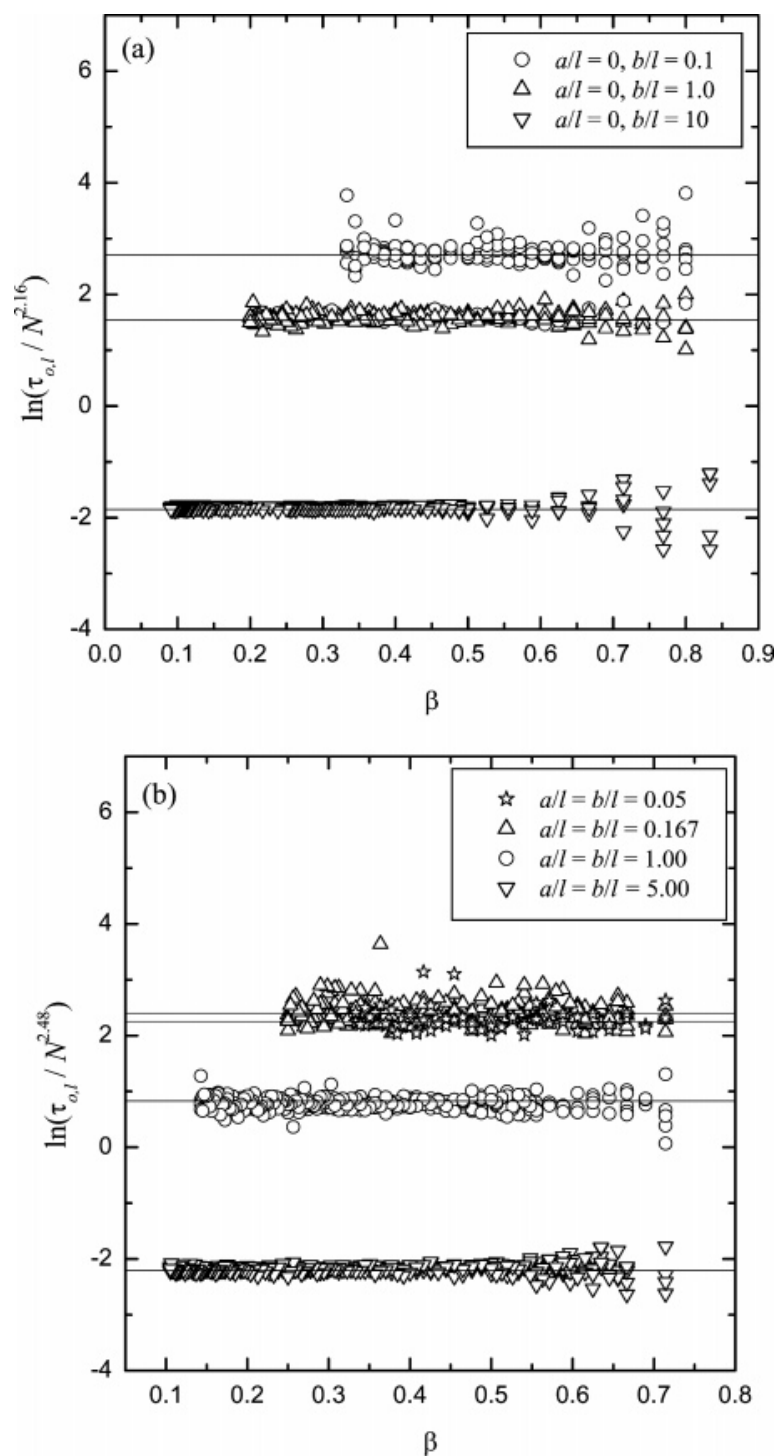

Figure 7. Variation of the rate constants $k_{o, l}$ with the temperature $\beta$ for different chain lengths: (a) end-to-interior, $\alpha \simeq 2.16$; (b) interior-to-interior, $\alpha \simeq 2.48$.

between reactive sites complicates the probability of loop formation. It is more difficult to form an interior-tointerior loop associated with a specified set of $\{a / l, b / l\}$ than the end-to-end loop, as long as the chain length is long enough. On the contrary, for a given chain length, the former can be more easily formed than the latter if the reactive sites are very close to each other (large $a / l$ and $b / l)$. This consequence indicates that biopolymers may utilize loop types and contour distance between reactive sites to control the probability of loop formation, which in turn influences its function. The typical persistence lengths of single-stranded and double-helix are around 8 bases and 100 base pairs, respectively. As a result, dsDNA with 1000 base pairs corresponds to about a flexible chain with 10 monomers. Our present analysis is applicable to ssDNA with $O\left(10^{2}\right)$ bases and dsDNA with $O\left(10^{3}\right)$ base pairs.

It should be emphasized that the simplicity of our model clearly illustrates the roles played by loop types and separation between two sites, but it also limits its relevance to realistic systems. Several factors may have important effects in DNA systems. For shorter chains, effects such as bending and stacking interactions come into play. ${ }^{8}$ For long DNA, the segment is more resem-
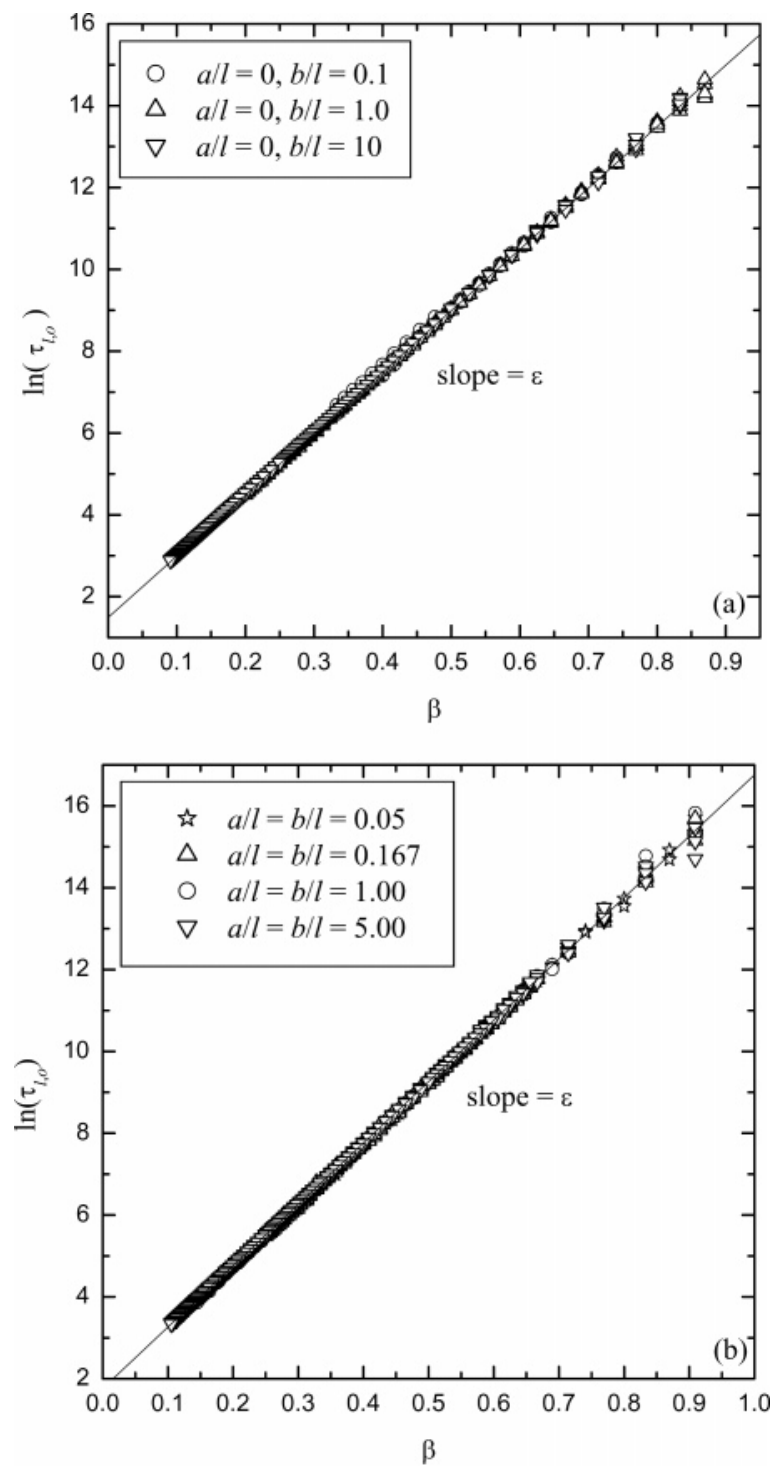

Figure 8. Variation of the rate constants $k_{l, o}$ with the temperature $\beta$ for different chain lengths: (a) end-to-interior; (b) interior-to-interior. The slope of the solid line is $\epsilon=15$.

bling rods than spheres. Large aspect ratios may be involved in thermodynamics of dsDNA coil-to-loop crossover, and thus effects due to relative orientation of statistical segments are likely to be important. A wormlike chain with excluded volume is more like the dsDNA molecule than a hard-sphere chain. Moreover, DNA in nature does not manifest itself as one single molecule. If more than one molecule is present, there is a possibility that reaction sites on different molecules stick together. ${ }^{4}$ The competition between loop and complex formation has been studied for a two polymers system by taking the zero temperature limit. ${ }^{9}$ It was observed that complex formation is preferred over loop formation at small values of $a / l$ or $b / l$.

Acknowledgment. This research is supported by the National Science Council of Taiwan, R.O.C.

\section{References and Notes}

(1) Goddard, N. L.; Bonnet, G.; Krichevsky, O.; Libchaber, A Phys. Rev. Lett. 2000, 85, 2400. Ansari, A.; Kuznetsov, S. V. Shen, Y. Proc. Natl. Acad. Sci. U.S.A. 2001, 98, 7771. Ying, L.; Wallace, M. I.; Klenerman, D. Chem. Phys. Lett. 2001, $334,145$. 
(2) Schlief, R. Annu. Rev. Biochem. 1992, 61, 199. Matthews, K. S. Microbiol. Rev. 1992, 56, 123 .

(3) Harmer, T.; Wu, M.; Schlief, R. Proc. Natl. Acad. Sci. U.S.A. 2001, 98, 427.

(4) Cary, R. B.; Peterson, S. R.; Wang, J.; Bear, D. G.; Bradbury, E. M.; Chen, D. J. Proc. Natl. Acad. Sci. U.S.A. 1997, 94, 4267.

(5) Wilemski, G.; Fixman, M. J. Chem. Phys. 1974, 60, 866 Szabo, A.; Schulten, K.; Schulten, Z. J. Chem. Phys. 1980 72, 4350. Pastor, R. W.; Zwanzig, R.; Szabo, A. J. Chem. Phys. 1996, 105, 3878 .
(6) Sheng, Y.-J.; Chen, J. Z. Y.; Tsao, H.-K. Macromolecules 2002 , 35, 9624. Sheng, Y.-J.; Lin, H.-J.; Chen, J. Z. Y.; Tsao, H.-K. J. Chem. Phys. 2003, 118, 8513.

(7) Des Cloizeaux, J.; Jannink, G. Polymers in Solution: Their Modeling \& Structure; Oxford University Press: Oxford, 1990; Chapter 13.

(8) Chen, J. Z. Y.; Tsao, H.-K.; Sheng, Y.-J. Europhys. Lett. 2004, $65,407$.

(9) Baljon-Haakamn, A. R. C.; Witten, T. A. Macromolecules 1992, 25, 2969.

MA049388Z 